\title{
Simple screening method for the diagnosis of nonB- nonc hepatocellular carcinoma
}

\author{
Kazuhiro Nouso1,2, Yoshie Furubayashi', Shohei Shiota', Akiko Wakuta', Ayano Oonishi', Kazuya \\ Kariyama ${ }^{1}$, Yasuto Takeuchi ${ }^{2}$, Nozomu Wada ${ }^{2}$, Hideki Onishi' ${ }^{2}$, Takuya Adachi ${ }^{2}$, Atsushi Oyama ${ }^{2}$, Chihiro \\ Dohi $^{2}$, Tetsuya Yasunaka ${ }^{2}$, Yuki Yasunaka ${ }^{2}$, Fusao lkeda $^{2}$, Hidenori Shiraha ${ }^{2}$, Akinobu Takaki ${ }^{2}$, Hiroyuki \\ Okada $^{2}$
}

'Department of Gastroenterology, Okayama City Hospital, Okayama 700-8557, Japan.

2Department of Gastroenterology and Hepatology, Okayama University Graduate School of Medicine, Dentistry and Pharmaceutical Sciences, Okayama 700-8558, Japan.

Correspondence to: Dr. Kazuhiro Nouso, Department of Gastroenterology, Okayama City Hospital, 3-20-1, Kitanagase-omotemachi, Kita-ku, Okayama City, Okayama 700-8557, Japan. E-mail: kazunouso@gmail.com

How to cite this article: Nouso K, Furubayashi Y, Shiota S, Wakuta A, Oonishi A, Kariyama K, Takeuchi Y, Wada N, Onishi H, Adachi T, Oyama A, Dohi C, Yasunaka T, Yasunaka Y, Ikeda F, Shiraha H, Takaki A, Okada H. Simple screening method for the diagnosis of nonBnonC hepatocellular carcinoma. Hepatoma Res 2018;4:73. http://dx.doi.org/10.20517/2394-5079.2018.93

Received: 8 Aug 2018 First Decision: 31 Aug 2018 Revised: 8 Nov 2018 Accepted: 9 Nov 2018 Published: 12 Dec 2018

Science Editor: Guang-Wen Cao Copy Editor: Cui Yu Production Editor: Huang-Liang Wu

\begin{abstract}
Aim: The incidence of non-virus-related nonB-nonC hepatocellular carcinoma (NBNC-HCC) is on the rise. However, screening at-risk individuals using imaging methods is complicated by the large size of the at-risk patient pool. The aim of this study is to develop an effective simple screening method, using blood tests.
\end{abstract}

Methods: The diagnostic value of aspartate aminotransferase (AST), alpha-fetoprotein (AFP), and des-gammacarboxy prothrombin (DCP) was analyzed using sera from 203 NBNB-HCC patients and 106 diabetes mellitus patients.

Results: Areas under receiver operating characteristic curves for AST, AFP, and DCP were $0.844,0.901$, and 0.914, respectively. The optimal cut-offs for diagnosing NBNC-HCC based on Youden indices were $30 \mathrm{IU} / \mathrm{L}$, $3.6 \mathrm{ng} / \mathrm{mL}$, and $25 \mathrm{mAU} / \mathrm{mL}$, respectively. On selecting patients who were positive at least one parameter (AST, AFP, or DCP), the sensitivity was $97.5 \%$. This high sensitivity was preserved (98.0\%) even in cases of nonadvanced $\mathrm{HCC}(\leq 3 \mathrm{~cm}, \leq 3$ nodules). Specificity was $72.6 \%$.

Conclusion: This simple triple screen for AST, AFP, and DCP appears to have diagnostic value in NBNC-HCC and could be used to select candidates for further testing using imaging.

Keywords: Hepatocellular carcinoma, screening, diagnosis, alpha-fetoprotein, aspartate aminotransferase, desgamma-carboxy-prothrombin 


\section{INTRODUCTION}

Hepatocellular carcinoma (HCC), a frequent complication of chronic hepatitis virus infection, is the secondleading cause of cancer death worldwide ${ }^{[1]}$. Due to the development of a HCC screening system with ultrasonography in patients with viral hepatitis, early diagnosis of HCC has been improved in $\operatorname{Japan}^{[2,3]}$. Moreover, the spread of direct acting antivirals for hepatitis $\mathrm{C}$ virus and nucleoside analogues for hepatitis $\mathrm{B}$ virus, has curtailed the incidence of hepatitis virus-related $\mathrm{HCC}^{[4-6]}$.

In contrast, the incidence of non-virus-related nonB-nonC HCC (NBNC-HCC) is on the rise ${ }^{[7]}$. Aging, excessive alcohol consumption, diabetes mellitus (DM), and non-alcoholic steatohepatitis (NASH) are considered major risk factors for NBNC-HCC ${ }^{[8,9]}$. However, periodic screening of patients at risk using imaging modalities, which is the recommended practice by the Japan society of hepatology ${ }^{[9]}$, is not realistic due to the large number of patients with these risk factors ${ }^{[10]}$. Even if screening were limited to only the patients with DM, the estimated patient number in Japan would be over 7 million $^{[1]]}$. In order to implement a comprehensive screening for NBNC-HCC, the development of effective non-imaging screening methods is necessary.

There are several reports indicating that an elevation in the serum transaminase levels is a risk factor for $\mathrm{HCC}^{[12,13]}$. In addition, serum levels of alpha-fetoprotein (AFP) were found to reliably predict the development of HCC in patients with chronic hepatitis $C$ virus infection who had achieved a sustained virological response ${ }^{[14-16]}$. A third potential biomarker, des-gamma-carboxy prothrombin (DCP) should also be considered as it was reported to be a better marker for HCC than AFP in NBNC-HCC ${ }^{[17]}$.

In this study, we examined the diagnostic utility of aspartate aminotransferase (AST), AFP, and DCP as non-imaging screening markers for NBNC-HCC.

\section{METHODS}

\section{Patients}

Between January 2001 and December 2016, 1,285 consecutive patients were initially diagnosed with HCC and treated at the Okayama University Hospital. Among these, 203 patients who were negative for both the hepatitis B virus surface antigen and hepatitis C virus antibody were diagnosed with NBNC-HCC and enrolled to the test group. Additionally, 106 patients with DM treated at the outpatient clinic of Okayama City Hospital were enrolled to the control group. For validation, 86 NBNC-HCC patients treated at Okayama City Hospital were also enrolled.

\section{Diagnosis}

HCC was diagnosed using imaging modalities such as computed tomography (CT), magnetic resonance imaging (MRI), or angiography. Diagnostic criteria for HCC were based on previous reports of hyperattenuation at the arterial phase or hypoattenuation at the portal phase, determined with dynamic CT or MRI, and tumor staining in angiography ${ }^{[18]}$.

There was no history of cancers, including HCC, in the control group, and abdominal ultrasonography was used to rule out HCC in the 6 months prior to enrollment. None of the patients were on warfarin or vitamin K.

\section{Determination of diagnostic accuracy}

Serum levels of AST, AFP, and DCP were compared between the NBNC-HCC and control groups. Sensitivity and specificity for the three markers used in the diagnosis of HCC were analyzed at different cut-offs. In addition, optimal cut-offs were determined using the receiver operating characteristic (ROC) curve and by calculation of Youden index. The rate of patients whose serum levels for any of the three markers were higher than the optimal cut-offs, was also analyzed. 
Table 1. Patient characteristics

\begin{tabular}{lccc}
\hline Variables & NBNC-HCC $(\boldsymbol{n}=\mathbf{2 0 3})$ & DM $(\boldsymbol{n}=\mathbf{1 0 6})$ & $\boldsymbol{P}$-value \\
\hline Age (years) & $69(24-90)$ & $65(25-92.0)$ & 0.024 \\
Sex (male) & $158(77.8 \%)$ & $64(60.4 \%)$ & 0.001 \\
Total bilirubin $(\mathrm{mg} / \mathrm{dL})$ & $0.8(0.1-4.8)$ & $0.6(0.2-1.2)$ & $<0.001$ \\
Albumin $(\mathrm{g} / \mathrm{dL})$ & $3.8(2.0-5.1)$ & $4.1(3.1-5.0)$ & $<0.001$ \\
Platelet $\left(\times 10^{4} / \mathrm{mm}^{3}\right)$ & $13.7(2.2-65.3)$ & $23.5(2.6-40.6)$ & $<0.001$ \\
AST $(\mathrm{IU} / \mathrm{L})$ & $42(14-611)$ & $22(13-75)$ & $<0.001$ \\
ALT $(\mathrm{IU} / \mathrm{L})$ & $33(2-377)$ & $20(9-247)$ & $<0.001$ \\
Tumor size $(\mathrm{mm})$ & $28(8-200)$ & $\mathrm{NA}$ & $\mathrm{NA}$ \\
Tumor number $(>3)$ & $53(26.4 \%)$ & $\mathrm{NA}$ & $\mathrm{NA}$ \\
AFP $(\mathrm{ng} / \mathrm{mL})$ & $8.5(0.6-376210)$ & $1.9(0.5-10.9)$ & $<0.001$ \\
DCP $(\mathrm{mAU} / \mathrm{mL})$ & $98(11-1323600)$ & $16(8-48)$ & $<0.001$ \\
\hline
\end{tabular}

Values are the median (range), unless otherwise indicated; NBNC-HCC: nonB-nonC hepatocellular carcinoma; DM: diabetes mellitus; AST: aspartate aminotransferase; ALT: alanine aminotransferase; AFP: alpha-fetoprotein; DCP: des-gamma-carboxy prothrombin; NA: not applicable

\section{Statistical analysis}

The baseline characteristics were summarized as medians and ranges. Differences in the continuous variables were compared using the Wilcoxon rank-sum test, while categorical variables were analyzed using the chi-square test. All significance tests were two-sided, and $P<0.05$ was considered significant. Analyses were performed using the JMP software program (ver. 13.0, SAS Institute Japan Ltd., Tokyo, Japan).

\section{RESULTS}

\section{Patient characteristics}

The patient characteristics are shown in Table 1. Patients in the NBNC-HCC group compared with the control group, respectively, were slightly older (median age, $69 v$ s. 65 years; $P=0.024$ ), with a higher percentage of males $(77.8 \%$ vs. $60.4 \%$; $P=0.001)$. The median tumor size was $28 \mathrm{~mm}$ and in $48.8 \%$ of cases, the tumor was under $3 \mathrm{~cm}$ in diameter with less than or equal to three nodules, and thus treatable by surgical resection or local ablation therapies. AST, AFP, and DCP were significantly elevated in the NBNC-HCC group compared to the control group.

\section{Evaluation of diagnostic accuracy of AST, AFP, and DCP}

Area under ROC (AUROC) curve values for AST, AFP, and DCP were 0.844 (95\% confidence interval; 0.7930.884), 0.901 (95\% confidence interval; 0.861-0.929), and 0.914 (95\% confidence interval; 0.878-0.940), respectively. The optimal cut-off values, as calculated with Youden indexes, were $30 \mathrm{IU} / \mathrm{L}, 3.6 \mathrm{ng} / \mathrm{mL}$, and $25 \mathrm{mAU} /$ $\mathrm{mL}$, respectively. Positivity rates for the different parameters in the two groups at different cut-offs are shown in Table 2. The combination of the three factors with the optimal cut-offs achieved a high positive rate (97.5\%) in the NBNC-HCC group [Figure 1A], which was maintained (98.0\%) in a subgroup of patients with non-advanced HCC ( $\leq 3 \mathrm{~cm}, \leq 3$ nodules). Using the same cut-offs, the positive rate in the control group was $27.4 \%$ [Figure 1B].

Because the control group consisted of patients with DM, we also analyzed the positive rate in NBNC-HCC patients with DM. The rates were 98.5\% (66/77) in all HCC patients with DM and 97.0\% (32/33) in patients with DM with non-advanced HCC ( $\leq 3 \mathrm{~cm}, \leq 3$ nodules). The test positivity rates in NBNC-HCC patients without DM were 97.1\% (132/136; all cases) and 98.5\% (64/65; non-advanced HCC). No statistical difference in the positive rate was observed when comparing NBNC-HCC cases with and without DM $(P=0.531)$.

We checked the positivity in the validation set and similar result was obtained (81/86, 94.2\%). The rate was maintained $(61 / 57,93.4 \%)$ in non-advanced HCC $(\leq 3 \mathrm{~cm}, \leq 3$ nodules). 
Table 2. Diagnostic accuracy using different cut-offs

\begin{tabular}{|c|c|c|c|c|}
\hline \multirow[t]{2}{*}{ Variables (cut-off) } & & \multicolumn{3}{|c|}{ Positive rate } \\
\hline & & NBNC-HCC & $\leq \mathbf{3} \mathrm{cm}, \leq 3$ nodules & DM \\
\hline \multirow[t]{3}{*}{ AFP } & $(3.6 \mathrm{ng} / \mathrm{mL})^{\star}$ & $161(79.3 \%)$ & $74(75.5 \%)$ & $10(9.4 \%)$ \\
\hline & $(5 \mathrm{ng} / \mathrm{mL})$ & $138(68.0 \%)$ & $56(57.1 \%)$ & $5(4.7 \%)$ \\
\hline & $(10 \mathrm{ng} / \mathrm{mL})$ & $96(47.3 \%)$ & $29(29.6 \%)$ & $1(0.94 \%)$ \\
\hline \multirow[t]{3}{*}{ DCP } & $(25 \mathrm{mAU} / \mathrm{mL})^{\star}$ & $165(81.3 \%)$ & $71(72.5 \%)$ & $9(8.5 \%)$ \\
\hline & $(40$ mAU/mL) & $144(70.9 \%)$ & $53(54.1 \%)$ & $1(0.94 \%)$ \\
\hline & $(100 \mathrm{mAU} / \mathrm{mL})$ & $101(49.8 \%)$ & $25(25.5 \%)$ & 0 \\
\hline \multirow[t]{3}{*}{ AST } & $(30 \mathrm{IU} / \mathrm{L})^{\star}$ & $148(72.9 \%)$ & $66(67.4 \%)$ & $16(15.1 \%)$ \\
\hline & $(40 \mathrm{IU} / \mathrm{L})$ & $107(52.7 \%)$ & $45(45.1 \%)$ & $10(9.4 \%)$ \\
\hline & $(80 \mathrm{IU} / \mathrm{L})$ & $24(11.8 \%)$ & $6(6.1 \%)$ & 0 \\
\hline Combination ${ }^{\star \star}$ & & $198 / 203(97.5 \%)$ & $96 / 98(98.0 \%)$ & $29 / 106(27.4 \%)$ \\
\hline $\mathrm{HCC}$ with diabetes & & $66 / 67(98.5 \%)$ & $32 / 33(97.0 \%)$ & - \\
\hline $\mathrm{HCC}$ without diabetes & & $132 / 136(97.1 \%)$ & $64 / 65(98.5 \%)$ & - \\
\hline
\end{tabular}

*Optimum value for detecting HCC; ${ }^{* *}$ combination of AST, AFP, and DCP; NBNC-HCC: nonB-nonC hepatocellular carcinoma; DM: diabetes mellitus; AFP: alpha-fetoprotein; DCP: des-gamma-carboxy prothrombin; AST: aspartate aminotransferase; HCC: hepatocellular carcinoma
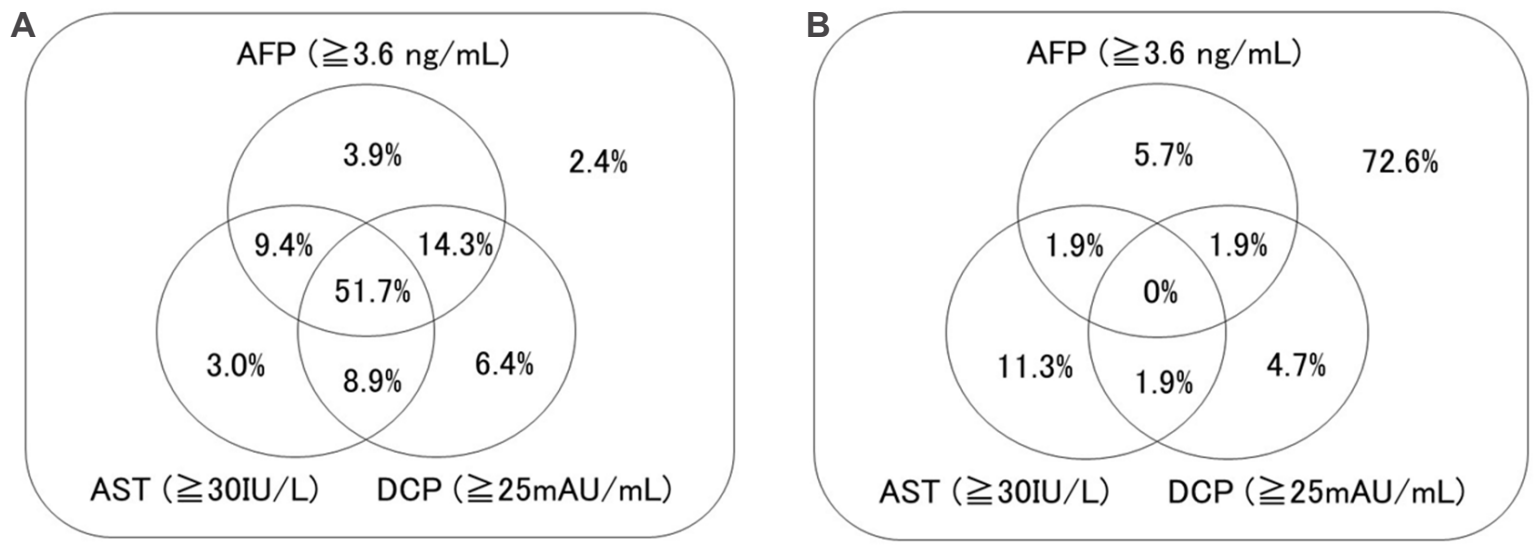

Figure 1. Distribution of marker-positive patients. Distribution of the patients positive for aspartate aminotransferase (AST), alphafetoprotein (AFP), and des-gamma-carboxy prothrombin (DCP) in patients with nonB-nonC hepatocellular carcinoma (NBNC-HCC). The majority (97.6\%) of patients with NBNC-HCC showed elevations in AST, AFP and/or DCP over the selected cut-offs (A); distribution of the three markers in patients with diabetes mellitus (B). One-fourth (27.4\%) of the patients showed elevations in AST, AFP, and/or DCP over the selected cut-offs

\section{DISCUSSION}

This study shows, for the first time, that a triple screen for serum AST, AFP, and DCP can be used to identify patients at high risk for NBNC-HCC. Over $95 \%$ of patients with NBNC-HCC in this study showed elevations in AST ( $\geq 30 \mathrm{IU} / \mathrm{L})$, AFP ( $\geq 3.6 \mathrm{ng} / \mathrm{mL})$ and/or DCP $(\geq 25 \mathrm{mAU} / \mathrm{mL})$, regardless of the diabetic status. In contrast, only one-fourth (27.4\%) of DM patients without HCC showed elevations in AST, AFP, and/or DCP. These patients can be considered to be at risk for developing HCC, meaning that this triple screening method could be used to identify patients who need further testing using imaging.

AFP is an oncofetal protein originally recognized as an HCC tumor marker ${ }^{[19]}$. There are many studies that set the cut-offs at high level because most of the control subjects were active chronic viral hepatitis. As the results, many of them showed low diagnostic abilities. Its sensitivity in HCC diagnosis increased to range between $49 \%$ and $71 \%$ when using a lower cut-off of $20 \mathrm{ng} / \mathrm{mL}^{[9]}$. However, AFP has not been considered a tumor marker of choice in NBNC-HCC, as its elevation in this condition is less pronounced than that in hepatitis virus-related $\mathrm{HCC}^{[17]}$. Recently, AFP has also been identified as a marker of carcinogenic potential of the liver. By using low cut-off $(5 \mathrm{ng} / \mathrm{mL})$, it can reliably predict development of HCC in chronic hepatitis C 
patients who have achieved a sustained virological response ${ }^{[14-16]}$. The positive rate in DM patients was only $4.7 \%$, using the same cut-off level. The rate was still low (9.4\%) even when the cut-off was lowered to $3.6 \mathrm{ng} / \mathrm{mL}$, which is the cut-off adopted in this study. Approximately $80 \%$ of patients with NBNC-HCC showed an AFP elevation over $3.6 \mathrm{ng} / \mathrm{mL}$. These results indicate that AFP is a good marker for NBNC-HCC with the low cutoff, in agreement with the results obtained in HCV patients with a sustained virological response. It should be noted that the origin of serum AFP, whether from highly carcinogenic liver parenchymal cells or from HCC, remains unclear.

In contrast to AFP, DCP is considered a reliable marker for NBNC-HCC, and was hence included in this combination screen ${ }^{[17]}$. The sensitivity and specificity of DCP at a commonly used cut-off $(40 \mathrm{mAU} / \mathrm{mL})$ were $70.9 \%$ and $99.1 \%$, respectively. Even on lowering the cut-off to $25 \mathrm{mAU} / \mathrm{mL}$ to maximize the detection rate, we still observed a high specificity (91.5\%). Although DCP testing is not meaningful in patients using drugs affecting vitamin $\mathrm{K}$ levels (warfarin, menatetrenone, etc.), our results show that it is a good marker for NBNC-HCC in the majority of cases.

Several reports indicate that an elevation in serum transaminase levels increases the risk for $\mathrm{HCC}^{[12,13]}$. A large cohort study examining over 0.4 million people conducted by a private health screening firm in Taiwan revealed that abnormal AST levels were associated with a 3.3-10.9-fold increased risk for HCC, compared with normal AST levels $(<25 \mathrm{IU} / \mathrm{L})^{[13]}$. In contrast, hazard ratio of the patients with abnormal alanine aminotransferase (ALT) levels was not high (1.29). In preliminary analysis with our cohort, the AUROC for ALT (0.68) was significantly lower than that of AST $(0.84)(P<0.001)$. Based on these findings, we included AST in the triple screen.

In this study, we show that the use of a triple screening method increased the sensitivity for HCC to $97.5 \%$. Although the specificity was decreased to $72.6 \%$, this index is useful because it reduced the number of candidates who required further screening to one-fourth of the original population size, which is a more manageable number for screening with imaging. Notably, this screening strategy produced high sensitivity even in patients in the early stages of HCC (tumor size $\leq 3 \mathrm{~cm}, \leq 3$ nodules), indicating that a triple screen for AST, ALT, and DCP may be useful for early detection of HCC.

We also examined the effect of occult hepatitis B virus infection that might correlate with the development of NBNC-HCC. Among 203 NBNC-HCC, 68 (33.5\%) patients were positive for hepatitis B core antibody (HBc-Ab). However, no difference of the positivity of AFP, DCP and AST was observed between the patients with and without $\mathrm{HBc}-\mathrm{Ab}$ in this cohort (data not shown).

This study has several limitations. First, we could not effectively select the patients with NASH who need further examination with imaging. The patients with NASH often showed deterioration in liver function, which resulted in high AST and/or AFP levels in about 50\% of cases. Second, the cut-offs adopted for AST, ALT, and DCP are optimized for each individual marker, but when used in combination, may not optimally delineate the high-risk populations. While the usage of the formula obtained by logistic regression analysis produced a high AUROC (0.971), the calculation was too complex for use in the clinical setting. Furthermore, no data of healthy control was presented although it might strength the conclusion of this study. Given that this is a retrospective case study, another limitation is that use of the triple screen in this population cannot predict the actual risk for developing HCC, but merely has diagnostic ability. Prospective periodic measurement of these markers is required for early detection of HCC.

In conclusion, the screening method developed in this study is easy to use because it is a blood test consisted of AST, AFP and DCP. This study clearly showed that being aware of the new low-cut offs of the markers when we conduct blood tests with any reasons was important in achieving early diagnosis of NBNC-HCC. Although it is necessary to use imaging as a confirmatory test for NBNC-HCC, the use of this triple screen 
would reduce the number of patients requiring screening by imaging to one-fourth the original number. Further analysis in a large cohort is required to validate this screening method.

\section{DECLARATIONS}

\section{Acknowledgements}

We thank Wako Pure Chemical Industries for measuring tumor markers in patients with diabetes mellitus.

\section{Authors' contributions}

Design of the work, data analysis, interpretation and preparation of this manuscript: Nouso K

Data collection, data update and acquisition of serum samples: Furubayashi Y

Data collection, database construction: Shiota S, Wakuta A, Oonishi A, Kariyama K, Takeuchi Y, Wada N, Onishi H, Adachi T, Oyama A, Dohi C, Yasunaka T, Yasunaka Y, Ikeda F, Shiraha H, Takaki A

Gave advise for conducting the whole work: Okada $\mathrm{H}$

\section{Availability of data and materials}

The data will not be shared because the patients did not give their consents for the data to be analyzed by the third party.

\section{Financial support and sponsorship}

Wako Pure Chemical Industries measured tumor markers in patients with diabetes mellitus.

\section{Conflict of interest}

All authors declared that there are no conflicts of interest.

\section{Ethical approval and consent to participate}

All HCC patients provided written informed consent to use their clinical records for this study. In addition, written informed consent was obtained from the control group patients for tumor marker (AFP and DCP) measurement and use of clinical data. The study protocol conformed to the tenets of the Declaration of Helsinki and was approved by the institute ethics committees.

\section{Consent for publication}

Not applicable.

\section{Copyright}

(c) The Author(s) 2018.

\section{REFERENCES}

1. El-Serag HB. Epidemiology of viral hepatitis and hepatocellular carcinoma. Gastroenterology 2012;142:1264-73.

2. Miyahara K, Nouso K, Yamamoto K. Image of the month. Hepatocellular carcinoma mimicking bile duct stone. Clin Gastroenterol Hepatol 2010;8:e17.

3. Kudo M, Izumi N, Sakamoto M, Matsuyama Y, Ichida T, et al. Survival analysis over 28 years of 173,378 patients with hepatocellular carcinoma in Japan. Liver Cancer 2016;5:190-7.

4. Hosaka T, Suzuki F, Kobayashi M, Seko Y, Kawamura Y, et al. Long-term entecavir treatment reduces hepatocellular carcinoma incidence in patients with hepatitis B virus infection. Hepatology 2013;58:98-107.

5. Miyake Y, Iwasaki Y, Yamamoto K. Meta-analysis: reduced incidence of hepatocellular carcinoma in patients not responding to interferon therapy of chronic hepatitis C. Int J Cancer 2010;127:989-96.

6. Sung JJ, Tsoi KK, Wong VW, Li KC, Chan HL. Meta-analysis: treatment of hepatitis B infection reduces risk of hepatocellular carcinoma. Aliment Pharmacol Ther 2008;28:1067-77.

7. Tateishi R, Okanoue T, Fujiwara N, Okita K, Kiyosawa K, et al. Clinical characteristics, treatment, and prognosis of non-B, non-C hepatocellular carcinoma: a large retrospective multicenter cohort study. J Gastroenterol 2015;50:350-60.

8. Archambeaud I, Auble H, Nahon P, Planche L, Fallot G, et al. Risk factors for hepatocellular carcinoma in Caucasian patients with non-viral cirrhosis: the importance of prior obesity. Liver Int 2015;35:1872-6.

9. Kokudo N, Hasegawa K, Akahane M, Igaki H, Izumi N, et al. Evidence-based clinical practice guidelines for hepatocellular carcinoma: the 
Japan society of hepatology 2013 update (3rd JSH-HCC Guidelines). Hepatol Res 2015; doi: 10.1111/hepr.12464.

10. Forner A, Reig M, Bruix J. Hepatocellular carcinoma. Lancet 2018;391:1301-14.

11. International Diabetes Federation. IDF Diabetes Atlas - 8th edition. Available from: http://www.diabetesatlas.org. [Last accessed on 4 Dec 2018].

12. Ishiguro S, Inoue M, Tanaka Y, Mizokami M, Iwasaki M, et al. Serum aminotransferase level and the risk of hepatocellular carcinoma: a population-based cohort study in Japan. Eur J Cancer Prev 2009;18:26-32.

13. Wen CP, Lin J, Yang YC, Tsai MK, Tsao CK, et al. Hepatocellular carcinoma risk prediction model for the general population: the predictive power of transaminases. J Natl Cancer Inst 2012;104:1599-611.

14. Asahina Y, Tsuchiya K, Nishimura T, Muraoka M, Suzuki Y, et al. $\alpha$-fetoprotein levels after interferon therapy and risk of hepatocarcinogenesis in chronic hepatitis C. Hepatology 2013;58:1253-62.

15. Hiramatsu N, Oze T, Takehara T. Suppression of hepatocellular carcinoma development in hepatitis C patients given interferon-based antiviral therapy. Hepatol Res 2015;45:152-61.

16. Takeuchi Y, Ikeda F, Osawa T, Araki Y, Takaguchi K, et al. Alpha-fetoprotein before and after pegylated interferon therapy for predicting hepatocellular carcinoma development. World J Hepatol 2015;7:2220-8.

17. Ricco G, Cavallone D, Cosma C, Caviglia GP, Oliveri F, et al. Impact of etiology of chronic liver disease on hepatocellular carcinoma biomarkers. Cancer Biomark 2018;21:603-612.

18. Bruix J, Sherman M; American Association for the Study of Liver Diseases. Management of hepatocellular carcinoma: an update. Hepatology 2011;53:1020-2.

19. Tateishi R, Yoshida H, Matsuyama Y, Mine N, Kondo Y, et al. Diagnostic accuracy of tumor markers for hepatocellular carcinoma: a systematic review. Hepatol Int 2008;2:17-30. 